\title{
Pathology of Alcoholic Liver Disease
}

\author{
Romulo Celli ${ }^{1}$ and Xuchen Zhang $^{2}$ \\ ${ }^{1}$ Department of Pathology, Yale University School of Medicine, New Haven, CT, USA; ${ }^{2}$ Pathology and Laboratory Service, VA \\ Connecticut Health System and Department of Pathology, Yale University School of Medicine, West Haven, CT, USA
}

\begin{abstract}
Alcohol-attributable burden on global health is increasing, and the relationship between population alcohol consumption and liver-related deaths is strong. Longstanding scientific and clinical work has led to a relatively thorough, if not complete, understanding of the effects of alcohol consumption on the liver. Pathologic features of alcoholic liver disease (ALD) are recognized by pathologists and used to assist clinicians in diagnosing and determining severity of disease in patients suspected of ALD. In this review, we discuss the pathologic manifestations of ALD and provide salient points on their pathophysiology. In addition, the benefits and indications of liver biopsy and important differential diagnoses, including features distinguishing these entities, are reviewed.

(C) 2014 The Second Affiliated Hospital of Chongqing Medical University. Published by XIA \& HE Publishing Ltd. All rights reserved.
\end{abstract}

\section{Introduction}

There is a strong relationship between population alcohol consumption and liver-related deaths. In 2010, nearly 50\% of the world's population consumed alcohol in some form. During that year more than one million deaths worldwide were attributed to liver cirrhosis, and $47.9 \%$ of those were caused by chronic alcohol use. ${ }^{1}$

As a pathologic entity, alcoholic liver disease (ALD) can be defined as the manifold gross and microscopic manifestations of regular alcohol consumption on the liver. ALD is recognized as a progressive disease that worsens with chronic alcohol intake. Likewise, the pathological effects comprise a wide spectrum: from the banal and reversible steatosis to the severe and irreversible cirrhosis. The natural consequence of such phenotypic heterogeneity within a disease process is the presentation of certain pathologic features that are more specific for ALD (i.e. Central Hyaline Sclerosis) than others

Keywords: Alcoholic liver disease; Steatosis; Steatohepatitis; Pathology. Abbreviations: ALD, alcoholic liver disease; $A S H$, non-alcoholic fatty liver disease; CK8/18, cytokeratins 8 and 18; EASL, European Association for the Study of the Liver; FFA, free fatty acids; $H \& E$, hematoxylin and eosin; $N A D H$, nicotinamide adenine dinucleotide; NAFLD, non-alcoholic fatty liver disease; $\mathrm{NASH}$, non-alcoholic steatohepatitis; PDGF, platelet-derived growth factor; ROS, reactive oxygen species; TGF- $\beta 1$, transforming growth factor beta 1 ; TIMP-1, tissue inhibitors of metalloproteinase-1; TNFR2, TNF receptor 2; VLDL, very lowdensity lipoprotein.

Received: 3 March 2014; Revised: 14 April 2014; Accepted: 16 April 2014 DOI of original article: 10.14218/JCTH.2014.00010.

Correspondence to: Xuchen Zhang, Pathology and Laboratory Service, VA Connecticut Health System and Yale University School of Medicine, 950 Campbel Ave. West Haven, CT 06516, USA. Tel: +1-203-932-5711, Fax: +1-203-937-4704, E-mail: xuchen.zhang@yale.edu (i.e. steatosis). The role of the pathologist is to assimilate the diverse morphologic data from a given liver biopsy and to clearly determine progression of the disease and, if possible, its etiology.

Specifically, the role of liver biopsy in ALD is 1) to corroborate clinical findings in establishing diagnosis and 2) to estimate disease severity using semi-quantitative tools of disease grade and stage.

The objective of this review is to survey the gross and microscopic features of ALD and, in doing so, to provide clinicians with a reference for the interpretation of liver biopsy pathology reports. Additionally, we review the benefits and limitations of obtaining a liver biopsy and provide a preliminary set of indications for this clinical test.

\section{Major pathologic features of ALD}

\section{Steatosis}

Steatosis is the abnormal accumulation of mono-/di-/triglycerides and fatty acids in hepatocytes in the form of lipid droplets. ${ }^{2}$ In normal lipid metabolism, plasma free fatty acids (FFA)-unbound or attached to lipoprotein particles-are shuttled to the liver and either oxidized for fuel at the hepatocyte mitochondrion or stored as triglycerides. Triglycerides are exported as VLDL particles or organized into lipid droplets and kept indefinitely within the hepatocyte. Sufficient accumulation of lipid droplets leads directly to hepatocellular damage. An increase in plasma FFA concentration is seen in non-alcohol and alcohol-induced steatosis and is a likely contributing mechanism to pathologic liver fat accumulation. ${ }^{3}$ In addition, alcohol-induced steatosis stimulates a series of pathophysiologic disturbances. The oxidation of ethanol, for example, reduces nicotinamide adenine dinucleotide (NADH), which suppresses the oxidative mechanism of the mitochondria. Ethanol has also been shown, primarily in cell culture and animal models, to stimulate hepatic lipogenesis via activation of transcription factors such as sterol response element binding proteins (SREBP's), which regulate the expression of genes involved in lipid biosynthesis. ${ }^{4}$

Steatosis is the earliest and most common finding in ALD and is reversed upon cessation of alcohol consumption. ${ }^{5}$ It is estimated that normal liver parenchyma consists of up to $5 \%$ lipids. Therefore, by convention, a hepatic lipid deposition greater than $5 \%$ is considered pathologic. ${ }^{6}$

Steatosis presents with distinct gross and histological characteristics. Grossly, diffuse involvement of the liver by steatosis appears as generalized enlargement of the liver with a yellow-tinged appearance that looks and feels "greasy" to the touch. Microscopically, steatosis begins in 
zone 3 (perivenular/centrilobular) and extends outwards with increasing severity. ${ }^{7}$ Steatosis can be classified into microvesicular (small-droplet) or macrovesicular (large-droplet) types. Of the two, microvesicular steatosis is less prevalent and is characterized by hepatocytes with centrally placed nuclei and small vesicular fat droplets located circumferentially around the nucleus. Macrovesicular steatosis is characterized by hepatocytes that contain nuclei displaced to one side of the cell by the large fat droplets (Fig. 1A). Rupture of fat-laden hepatocytes can give rise to the appearance of lipogranulomas-microscopic foci of inflammation around fat droplets (Fig. 1B). These typically are seen singly and are often dispersed throughout the parenchyma. Chronic inflammatory cells may be seen scattered within the lobules, but they should not be present in significant density due to steatosis alone. In general, these characteristics make steatosis a readily identifiable pattern, but they are highly non-specific as there are no features of ethanol-induced steatosis that can help distinguish it from non-alcoholic fatty liver disease (NAFLD).

Classically, steatosis is considered a risk factor for disease progression to steatohepatitis and other more serious complications of liver disease (cirrhosis, hepatocellular carcinoma). However, studies involving both humans and animal models have demonstrated that steatosis may progress directly to fibrosis or cirrhosis without the characteristic inflammatory changes of steatohepatitis. ${ }^{2}$ For this reason, pathologists should examine even purely steatotic livers for the presence/degree of fibrosis. This will lead to a more comprehensive assessment of a patient's particular risk for progression to cirrhosis and its sequelae.

\section{Steatohepatitis}

Steatohepatitis is a histologic pattern that can be seen in chronic ethanol users. It is characterized by a set of diagnostic features, which may present in different stages of severity. ${ }^{8}$ Diagnostic features of steatohepatitis are parenchymal inflammation, hepatocyte damage, and fibrosis. This histologic pattern can be caused by the direct effect of alcohol (ASH) or multiple non-alcohol related etiologies (nonalcoholic steatohepatitis, NASH). In general, these changes are seen in a perivenular distribution in the earliest form of the disease, and they extend throughout the lobules as the

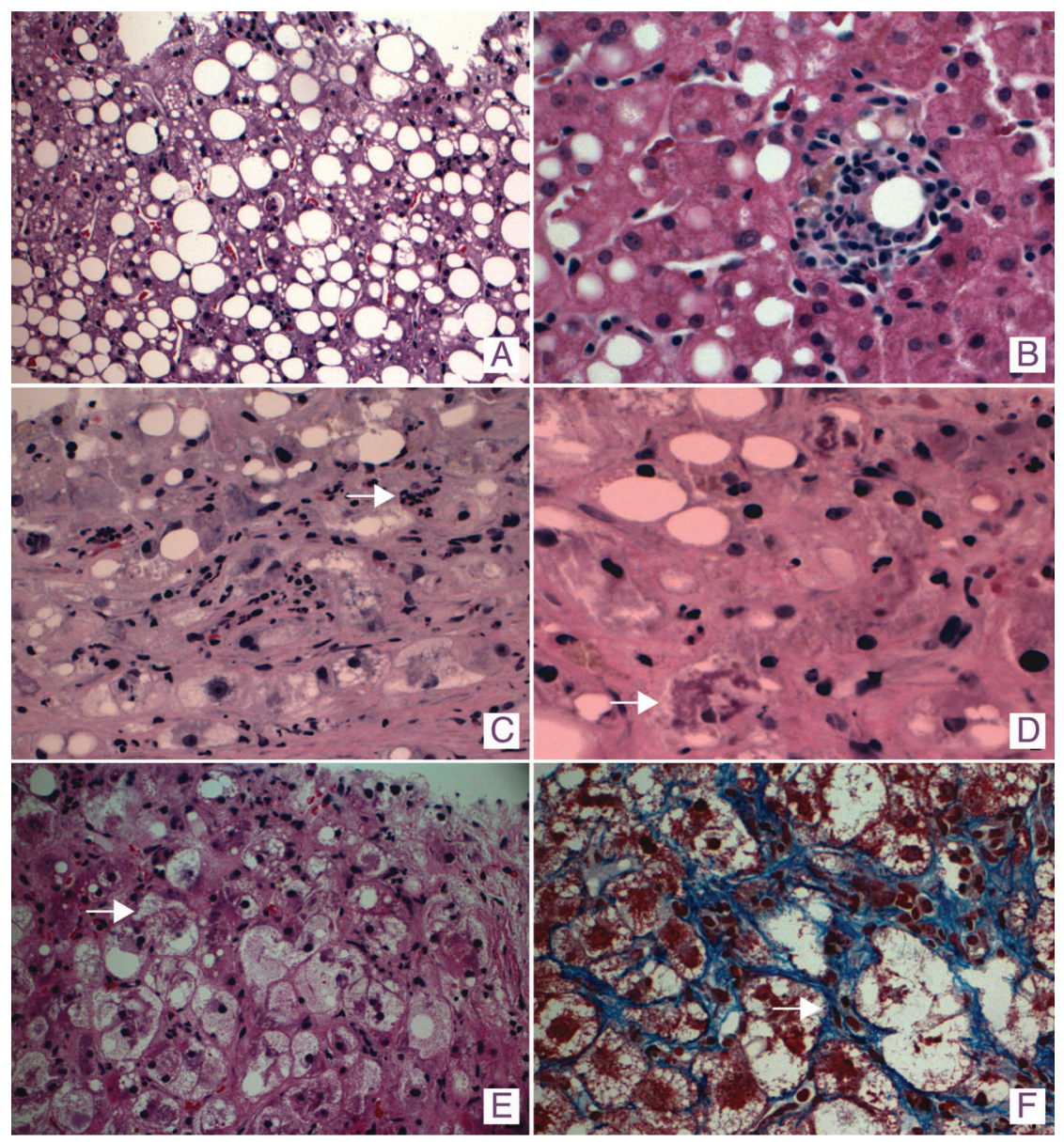

Fig. 1. 1A: Steatosis - Large-droplet (macrovesicular) and small-droplet (microvesicular) steatosis (200X, H\&E stain); 1B: Lipogranuloma - extracellular lipid surrounded by chronic inflammatory cells (200X, H\&E stain); 1C: Neutrophilic inflammation - Steatohepatitis with a predominantly neutrophilic inflammatory infiltrate (arrow showing clusters of neutrophils, 200X, H\&E stain); 1D: Mallory bodies - dense ropy eosinophilic skeins of cytokeratin filaments within cytoplasm of hepatocytes (arrow showing intracytoplasmic Mallory body, 400X, H\&E stain); 1E: Ballooning degeneration of hepatocytes - swollen and enlarged hepatocytes with an intracytoplasmic rarefied, "stringy" or "wispy" appearance (arrow showing hepatocytes with ballooning degeneration, 400X, H\&E stain); 1F: Perisinusoidal fibrosis - fibrosis in a "chicken-wire" pattern (arrow showing blue-colored perisinusoidal fibrosis, 400X, Trichrome stain) 
disease progresses. Despite similarities in nomenclature, the ASH histopathologic diagnosis can be seen in the chronic alcoholic with or without the acute clinical syndrome termed alcoholic hepatitis. In other words, ASH is not synonymous with alcoholic hepatitis, but patients with alcoholic hepatitis typically have $\mathrm{ASH}^{9}$

Inflammation in $\mathrm{ASH}$ has a varying amount of lobular involvement, with no specific zonal distribution. It is typically neutrophil-rich (Fig. 1C), ${ }^{8}$ although rarely it involves chronic inflammatory cells such as lymphocytes. Satellitosis can be present and appears as inflammatory cells encircling damaged hepatocytes. ${ }^{10}$ Neutrophilic infiltration has been attributed to the increase of chemokines, such as IL- 8 and IL17 , in both the serum and the liver parenchyma. ${ }^{11,12}$ It has been hypothesized that their function is to clear the liver of dying hepatocytes, and the baseline phagocytic function of these neutrophils has been reported to be decreased in human alcoholic patients. ${ }^{13}$ This could explain in part why infection is a major cause of mortality among patients with alcoholic hepatitis.

There are multiple morphologic manifestations of hepatocyte damage in ASH. Mallory-Denk bodies (also known as Mallory's hyaline, alcoholic hyaline, or Mallory bodies) are another classic, albeit non-specific finding, of alcoholic steatohepatitis. The presence of Mallory-Denk bodies reflects intracellular oxidative stress and can signal other liver pathology, including non-alcoholic steatohepatitis; Wilson disease; cholestatic conditions such as primary biliary cirrhosis; or exposure to certain drugs (such as amiodarone). They are characterized by ropy eosinophilic material within the hepatocyte cytoplasm (Fig. 1D), which is composed of misfolded and cross-linked cytokeratins 8 and $18\left(\right.$ CK8/18) ${ }^{14}$ as well as the ubiquitination-proteasome proteins ubiquitin, p62, heat-shock proteins (HSPs) 70 and 25, and other peptides. Immunohistochemical staining with $\mathrm{CK} 8 / 18$, ubiquitin, or p62 antibodies is useful for the identification of inconspicuous Mallory-Denk bodies. ${ }^{10}$ Of note, both CK8 and CK18 are able to bind to the TNF receptor 2 (TNFR2), thereby influencing TNF- $\alpha$-induced activation of apoptosis and neutrophilic inflammation through NF- $\kappa B$ activation. ${ }^{15}$ Thus, the accumulation of Mallory-Denk bodies may not simply be a byproduct of hepatocellular toxic damage but may also contribute to the perpetuation or advancement of inflammatory injury. ${ }^{16}$ Another invariably present finding that reflects cellular damage is hepatocyte "ballooning". Ballooned hepatocytes appear swollen or enlarged, with an intracytoplasmic rarefied "stringy" or "wispy" appearance (Fig. 1E). In contrast to steatotic hepatocytes, which have an entirely clear cytoplasm, ballooned cells have some wispy cytoplasmic content remaining. Apoptotic cells (also termed-apoptotic bodies or acidophilic bodies) are small hepatocytic cellular remnants with hyperchromatic condensed nuclei and a dense eosinophilic rim. They are often scattered in areas affected by inflammation. Apoptotic cells, ballooning hepatocytes, and Mallory-Denk bodies represent the hallmarks of hepatocyte damage in ASH.

The pattern of fibrosis in early ASH is often, although not exclusively, centrilobular. The presence of centrilobular/ perivenular fibrosis confers an increased risk for progression to cirrhosis. ${ }^{10,17}$ Hepatic stellate cells (previously Ito cells) under normal conditions have lipid-storing function and are responsible for producing the majority of intercellular collagen (fibrosis). This occurs following hepatic stellate cell activation by inflammation and hepatocyte damage (Fig. 3).
Activation of stellate cells leads to increase cell size and proliferation. These cells also gain the ability to destroy normal intercellular matrix and replace it with dense basement membrane-like collagen. Additionally, they increase the production of inflammatory cytokines, attracting more inflammatory cells and causing increased hepatocyte damage via a positive feedback mechanism. ${ }^{18}$ Light microscopic evidence of this process appears as dense collagen due to fibrosis. In particular, the process of fibrosis typically proceeds in a perisinusoidal and pericellular fashion, creating a distinctive "chicken-wire" pattern ${ }^{10}$ more easily seen with collagen stains such as trichrome or reticulin (Fig. 1F). These stains are particularly useful when pale hematoxylin and eosin (H\&E) stained sections make identification of collagen difficult.

In ASH, portal tracts may show small collections of lymphocytes, but portal tract findings should not be the most conspicuous feature of the biopsy. If larger, denser, and more frequent collections are seen, then other chronic hepatitides should be considered as the primary or concomitant pathology. ALD is strongly associated with other chronic hepatic diseases epidemiologically, especially hepatitis B and C. ${ }^{19,20}$ Comorbid hepatic diseases are common enough to warrant significant consideration each time a slide is examined.

\section{Steatofibrosis-cirrhosis}

There are multiple interplaying factors that contribute to the development of diffuse fibrosis-cirrhosis in ALD. Broadly, alcohol-induced hepatocyte damage leads to hepatic stellate cell activation. As discussed previously, activated stellate cells develop a myofibroblastic phenotype and the capacity to lay down basement-membrane like collagen. ${ }^{21}$ Thick collagen strands can be seen around the central vein as well as coursing through the hepatic lobules in a perisinusoidal and pericellular fashion, inhibiting diffusion of key nutrients to and from the hepatocytes and sinusoidal blood. This leads to starvation and focal atrophy of nearby hepatocytes. This, in turn, leads to an increase in scarring and thickening of the fibrous septa over time. Simultaneously, as a response to hepatic injury, there will be other areas of hepatocyte regeneration. The visible result of this interplay between fibrosis, hepatocyte atrophy, and focal regeneration is the appearance of cirrhotic nodules. These nodules can be classified as micronodular or macronodular depending on their size (larger or smaller than $3 \mathrm{~mm}$ ). Grossly, a liver that was once steatotic (enlarged and yellow/ brown in color) becomes cirrhotic (shrunken-often $<1 \mathrm{~kg}$ brown, firm, and nodular).

Although more likely to develop from ASH, fibrosis may also develop in the purely steatotic liver. ${ }^{16,22}$ In this scenario, fibrosis is the direct result of the injurious effects of alcohol, rather than secondary inflammation. This process typically takes longer than the more aggressive natural history of ASHinduced fibrosis. A separate and equally important point is that a cirrhotic liver may lose all of its fat content in the process of fibrosis. This increases the difficulty of determining etiology in an end stage liver.

\section{Other pathologic findings of ALD}

\section{Cholestasis}

Histologic cholestasis is more often seen in ASH than NASH and can be a key feature when distinguishing between these 
entities. Microscopically (Fig. 2A), cholestasis can take the form of inspissated bile-within canaliculi or intra-hepatocyte-termed bile "plugs" or "thrombi." Another feature of cholestasis is bile ductular proliferation. Also known as ductular reaction, this histologic feature is composed of an increase in bile ductular profiles within fibrotic portal tracts or along the fibrous septa of bridging fibrosis or cirrhosis. It is frequently accompanied by neutrophils. ${ }^{23}$ Cholestasis can be seen in all stages (steatosis, ASH, or cirrhosis) of ALD and ranges in severity. Its presence microscopically has been associated with worse prognosis in patients with clinical alcoholic hepatitis ${ }^{24}$ and histologic $\mathrm{ASH} .{ }^{25}$

\section{Alcoholic foamy degeneration}

Alcoholic foamy degeneration, originally described by Uchida et al in 1983, is an uncommon pattern of microvesicular steatosis (Fig. 2B), which has been described as classically centrilobular ${ }^{26}$ and at times diffuse. ${ }^{7}$ Clinically, the patient may present with acute hepatotoxicity and markedly elevated serum gamma-glutamyl transaminase levels, with or without elevation of the transaminases. ${ }^{26}$ The pathogenesis is related to mitochondrial dysfunction, and an identical histologic pattern can be seen in Reye's syndrome, tetracycline toxicity, and fatty liver of pregnancy. ${ }^{7,26}$

\section{Megamitochondria}

Megamitochondria represent eosinophilic sphere-like intracytoplasmic forms of this organelle on H\&E staining (Fig. 2C). ${ }^{27}$ While not specific for ALD, they provide a diagnostic clue, particularly when found in the center of the lobule. Their presence is related to the amount of daily ethanol use and to the length of abstinence before the time of biopsy. ${ }^{28}$

\section{Perivenular fibrosis-central hyaline sclerosis}

The criteria for the interpretation of perivenular fibrosis include extending at least two-thirds the perimeter of the terminal hepatic venule with the fibrous rim measuring over $4 \mu \mathrm{m}$ in thickness. ${ }^{17,29}$ In its extreme form, perivenular fibrosis can cause the necrosis of adjacent hepatocytes and fibrous thickening and obliteration of the central

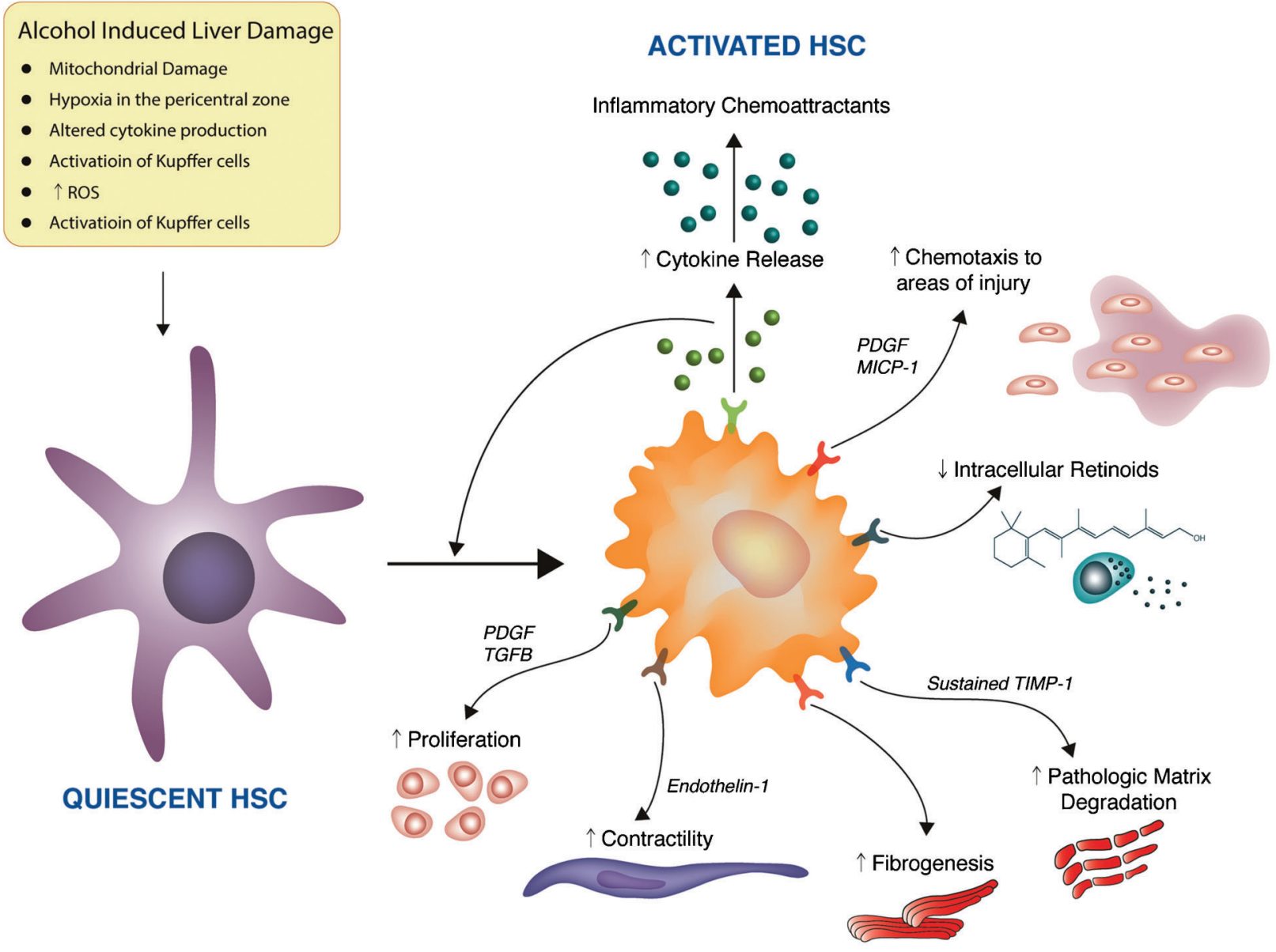

Fig. 2. Hepatic Stellate Cell (HSC) activation. Both alcohol and inflammation damage the liver and induce stellate cell activation. Apart from a phenotypic enlargement of the cell and nucleus, the stellate cells gain the capacity to degrade normal intercellular matrix and replace it with fibrous tissue (collagen). Additionally, they are able to proliferate and migrate to areas of injury, as well as attract more inflammatory cells, leading to further liver damage and HSC activation. ROS- reactive oxygen species; PDGF- platelet-derived growth factor; MCP-1- monocyte chemotactic protein-1; TGF- $\beta 1$ - Transforming growth factor beta 1 ; TIMP-1- tissue inhibitors of metalloproteinase-1. 


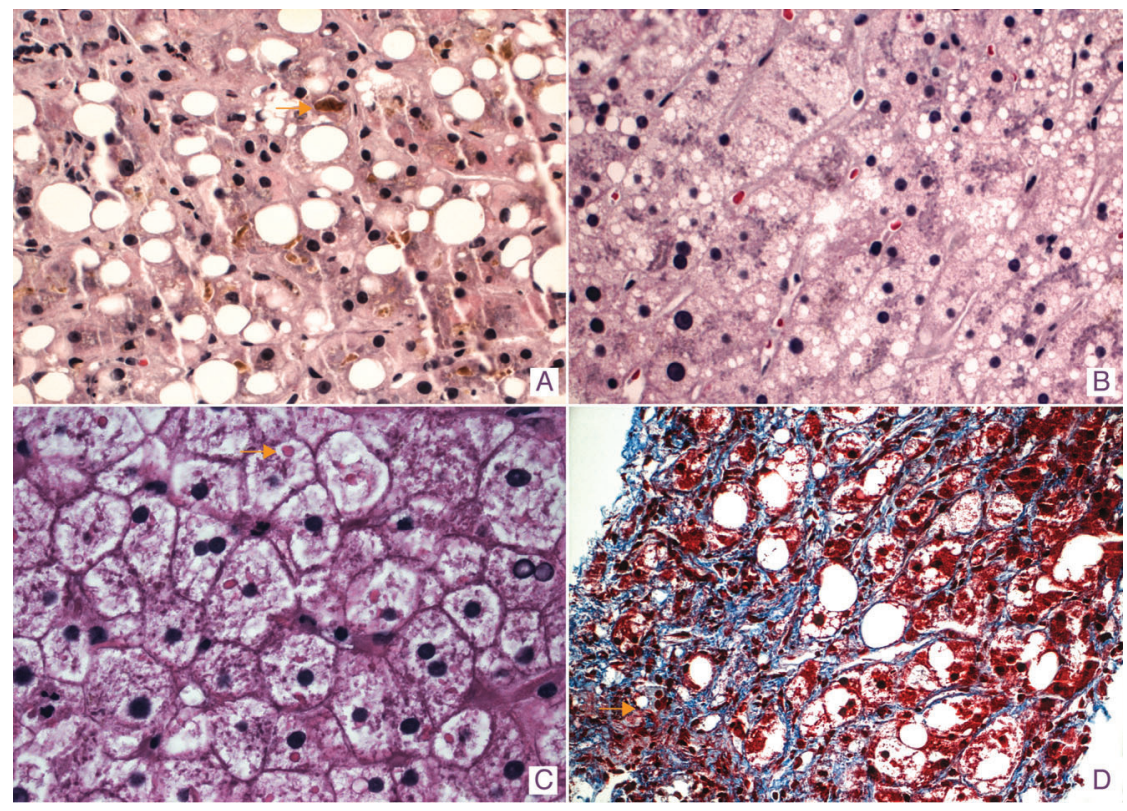

Fig. 3. 3A: Cholestasis - hepatocytic and canalicular cholestasis (arrow showing canalicular steatosis, 400X, H\&E stain); 3B: Foamy cell degeneration - enlarged hepatocytes with diffuse microvesicular steatosis (200X, H\&E stain); 3C: Megamitochondria - multiple eosinophilic sphere-like megamitochondria in the cytoplasm of hepatocytes (arrow showing megamitochondria in hepatocyte, 400X, H\&E stain); 3D: Central hyaline sclerosis - central venule with fibrosis and obliteration of its lumen (arrow showing obliterated central venule, 400X, trichrome stain).

venule-referred to as central hyaline sclerosis (Fig. 2D). ${ }^{30}$ When diffuse, this unique lesion may cause pre-cirrhotic portal hypertension. ${ }^{31}$

\section{Siderosis}

An iron stain (i.e. Prussian blue) reveals an increase in parenchymal iron in later stage ALD, particularly within Kupffer cells. Alcohol increases iron absorption in the gut. Significant siderosis, including significant iron staining within hepatocytes, should prompt consideration of a concurrent process such as hereditary hemochromatosis.

\section{Grading and staging of ALD}

As we have sought to demonstrate, there are many variables to scrutinize in the analysis of any liver biopsy. Consequently, a system that would standardize the scoring of these variables in concert for the purpose of disease staging and prognostication would be welcome. In the absence of a consensus method for semi-quantitative scoring of ALD severity, many pathologists have adopted the system proposed originally by Brunt et al. ${ }^{32}$ for use in NASH. In this system, the severity of parenchymal necroinflammatory activity is reflected by the grade of the lesion. Grade has been shown to correlate with patient AST and ALD levels. ${ }^{32}$ Mild activity (grade 1 ) represents steatosis involving up to $66 \%$ of lobules and mild steatohepatitis, while severe grade 3 represents panlobular steatosis and florid steatohepatitis. The severity of architectural distortions (fibrosis) is reflected by increasing stage. Stage 1 represents pericellular fibrosis (focal or extensive), while the higher stages 3 and 4 represent bridging fibrosis and cirrhosis, respectively. Biopsy is required for the diagnosis of histologic ALD or $\mathrm{ASH}$. In terms of staging fibrosis, novel non-invasive imaging and serum marker tests have been developed that correlate well with biopsy results. ${ }^{18,33}$

\section{Pathologic differential diagnosis}

The differential diagnoses of pathologic ALD depend on the specific parenchymal changes seen. For example, alcoholinduced macrovesicular steatosis is indistinguishable from the steatosis seen in the metabolic syndrome. The presence of microvesicular steatosis, which is less common and due to mitochondrial oxidative stress, raises a differential diagnosis which includes fatty liver of pregnancy, Reye's syndrome, and the effect of anti-retroviral therapies in AIDS patients. Clinical history and other relevant clinical data are essential in differentiating the causes of these similar patterns.

In the case of steatohepatitis, the most important differential diagnosis is NASH, itself an etiologically diverse entity. Besides clinical history, which may or may not be useful in distinguishing between these two frequent diseases, there are clues in the biopsy tissue that may suggest one etiology over the other. For example, in $\mathrm{ASH}$, necroinflammation is generally more prominent; meaning that there are more widespread neutrophilic infiltration, lipogranulomas, central hyaline sclerosis, and significantly increased density of Mallory-Denk bodies. Although the presence of MalloryDenk bodies in general is largely non-specific, a biopsy with a high enough density of these may be considered ASH until proven otherwise. Cholestasis is another feature that is more commonly present in ASH than NASH and may be used as a diagnostic clue. It is important to remember that a definitive diagnosis can only be made following acquisition of a thorough clinical history. ${ }^{34}$ Also, it is not uncommon that a patient may share these diseases, with morphologic overlap making their distinction nearly impossible. 
Celli R. et al.: Pathology of alcoholic liver disease

\section{Indication for liver biopsy in the setting of ALD}

With the advent of new technologies and other methods that allow for non-invasive evaluation of liver parenchyma, ${ }^{35}$ it is useful to critically review the role of liver biopsy in the setting of ALD. There are no precise indications for the use of this study, and this remains a source of active discussion. ${ }^{36}$ For example, some argue that liver biopsy is a diagnostic aid and not necessary in establishing the diagnosis or determining the prognosis of alcoholic hepatitis. Furthermore, they believe how much weight to put on the biopsy depends on the acuity of the clinical situation and the turn-around time of the particular histology lab. 9,36,37 Others argue that biopsy, in addition to the correct clinical context, is a key component of the diagnostic gold standard of alcoholic hepatitis. ${ }^{38}$ As part of their recently published clinical practical guidelines, the European Association for the Study of the Liver (EASL) acknowledged the lack of precise indications for biopsy in routine practice but advocated for its application in the following three specific scenarios: in patients with severe steatohepatitis to confirm diagnosis for initiation of treatment with corticosteroids and/or pentoxyphylline; in patients with other cofactors which may contribute to liver disease (in order to determine the relative contribution of each); and in clinical trials in order to follow patients' progress. ${ }^{39}$

Liver biopsy is not only diagnostic but also provides important long-term and treatment-related prognostic indicators. In our review of the literature, we found numerous scattered reports of proposed prognostic markers of biopsy, many of which are not validated and/or do not routinely show up on a pathology report. For example, a diagnosis of ASH or cirrhosis increases mortality by over $50 \%$ relative to fatty liver alone. ${ }^{40}$ Likewise, the amount of parenchymal neutrophilic infiltrate has been positively correlated with clinical response to corticosteroids. ${ }^{41}$

Notably, the liver biopsy should always be reserved for patients for whom the risk of hemorrhage is low; and the transjugular approach is favored in patients with hepatic coagulopathy.

\section{Conclusions}

Liver biopsy plays an important role in the real-time clinical assessment of patients. Tissue histology not only yields diagnostic information but also important information about the patient's overall disease progression. Clear and open communication between the pathologist and the treating clinician is key to successfully work through the oftentimes complex clinico-pathological data and to arrive at the best decisions (from microscope-to-bedside) for a given patient.

From steatosis to steatohepatitis and from Mallory bodies to megamitochondria-the morphologic heterogeneity of ALD is as varied as it is difficult to manage. It is only after decades of investigation by laboratory scientists and clinical physicians alike that the mechanisms of disease and the associations between microscopic morphology and clinical syndromes have started to come into focus. However, much work is left to be done. Future studies may take advantage of emerging molecular/genomic technologies in order to elucidate the clinically important markers of this disease. ${ }^{42}$ With time, better understanding should lead to better clinical outcomes.

\section{Conflict of interest}

None

\section{Author contributions}

Reviewing the literature and drafting the manuscript (RC), providing overall intellectual input into the design and editing the final version of the manuscript $(X Z)$, approval of the final version to be published $(\mathrm{RC}, \mathrm{XZ})$.

\section{REFERENCES}

[1] Rehm J, Samokhvalov AV, Shield KD: Global burden of alcoholic liver diseases. J Hepatol 2013;59:160-168. doi: 10.1016/j.jhep.2013.03.007.

[2] Mavrelis PG, Ammon HV, Gleysteen JJ, Komorowski RA, Charaf UK: Hepatic free fatty acids in alcoholic liver disease and morbid obesity. Hepatology 1983;3:226-231. doi: 10.1002/hep.1840030215.

[3] Bradbury MW: Lipid metabolism and liver inflammation I. Hepatic fatty acid uptake: possible role in steatosis. Am J Physiol Gastrointest Liver Physiol 2006;290:G194-198. doi: 10.1152/ajpgi.00413.2005.

[4] Ji C, Chan C, Kaplowitz N: Predominant role of sterol response element binding proteins (SREBP) lipogenic pathways in hepatic steatosis in the murine intragastric ethanol feeding model. J Hepatol 2006;45:717-724. doi: 10.1016/j.jhep.2006.05.009.

[5] Donohue TM, Jr.: Alcohol-induced steatosis in liver cells. World ] Gastroenterol 2007;13:4974-4978.

[6] Odze RD G]: Surgical Pathology of the GI Tract, Liver, Biliary Tract, and Pancreas: Saunders EISevier, 2009.

[7] Theise N: Histopathology of Alcoholic Liver Disease. Clin Liver Dis 2013;2: 64-67. doi: $10.1002 / \mathrm{cld} .172$.

[8] Alcoholic liver disease: morphological manifestations. Review by an international group. Lancet 1981;1:707-711.

[9] Lucey MR, Mathurin P, Morgan TR: Alcoholic hepatitis. N Engl J Med 2009, 360:2758-2769. doi: 10.1056/NEJMra0805786.

[10] Lefkowitch JH: Morphology of alcoholic liver disease. Clin Liver Dis 2005; 9 : 37-53. doi: 10.1016/j.cld.2004.11.001.

[11] Lemmers A, Moreno C, Gustot T, Marechal R, Degre D, Demetter P, de Nadai P, Geerts A, Quertinmont E, Vercruysse V, Le Moine O, Deviere J: The interleukin-17 pathway is involved in human alcoholic liver disease. Hepatology 2009;49:646-657. doi: 10.1002/hep.22680.

[12] McClain C], Barve S, Deaciuc I, Kugelmas M, Hill D: Cytokines in alcoholic liver disease. Semin Liver Dis 1999;19:205-219. doi: 10.1055/s-20071007110.

[13] Stadlbauer V, Mookerjee RP, Hodges S, Wright GA, Davies NA, Jalan R: Effect of probiotic treatment on deranged neutrophil function and cytokine responses in patients with compensated alcoholic cirrhosis. J Hepatol 2008;48:945-951. doi: 10.1016/j.jhep.2008.02.015.

[14] Stumptner C, Omary MB, Fickert P, Denk H, Zatloukal K: Hepatocyte cytokeratins are hyperphosphorylated at multiple sites in human alcoholic hepatitis and in a mallory body mouse model. Am J Pathol 2000;156:77-90. doi: 10.1016/S0002-9440(10)64708-6.

[15] Caulin C, Ware CF, Magin TM, Oshima RG: Keratin-dependent, epithelial resistance to tumor necrosis factor-induced apoptosis. J Cell Biol 2000;149: 17-22. doi: $10.1083 / \mathrm{jcb} .149 .1 .17$.

[16] Crawford JM: Histologic findings in alcoholic liver disease. Clinics in liver disease 2012;16:699-716. doi: 10.1016/j.cld.2012.08.004.

[17] Worner TM, Lieber CS: Perivenular fibrosis as precursor lesion of cirrhosis. JAMA 1985;254:627-630. doi: 10.1001/jama.1985.03360050065027.

[18] Vera M, Nieto N: Hepatic stellate cells and alcoholic liver disease. Rev Esp Enferm Dig 2006;98:674-684. doi: 10.4321/S1130-01082006000900005.

[19] Rosman AS, Waraich A, Galvin K, Casiano J, Paronetto F, Lieber CS: Alcoholism is associated with hepatitis $C$ but not hepatitis $B$ in an urban population. Am J Gastroenterol 1996;91:498-505.

[20] Caldwell SH, Jeffers L], Ditomaso A, Millar A, Clark RM, Rabassa A, Reddy KR, De Medina M, Schiff ER: Antibody to hepatitis $C$ is common among patients with alcoholic liver disease with and without risk factors. Am J Gastroenterol 1991;86:1219-1223.

[21] Friedman SL: Hepatic fibrosis - overview. Toxicology 2008;254:120-129. doi: $10.1016 /$ j.tox.2008.06.013.

[22] Lee UE, Friedman SL: Mechanisms of hepatic fibrogenesis. Best Pract Res Clin Gastroenterol 2011;25:195-206. doi: 10.1016/j.bpg.2011.02.005.

[23] Tung BY, Carithers RL, Jr.: Cholestasis and alcoholic liver disease. Clin Liver Dis 1999;3:585-601. doi: 10.1016/S1089-3261(05)70086-6. 
Celli R. et al.: Pathology of alcoholic liver disease

[24] Nissenbaum M, Chedid A, Mendenhall C, Gartside P: Prognostic-Significance of Cholestatic Alcoholic Hepatitis. Dig Dis Sci 1990;35:891-896. doi: 10.1007/BF01536804.

[25] Spahr L, Rubbia-Brandt L, Genevay M, Hadengue A, Giostra E: Early liver biopsy, intraparenchymal cholestasis, and prognosis in patients with alcoholic steatohepatitis. BMC gastroenterol 2011;11:115. doi: 10.1186/1471230X-11-115.

[26] Suri S, Mitros FA, Ahluwalia JP: Alcoholic foamy degeneration and a markedly elevated GGT: a case report and literature review. Dig Dis Sci 2003;48: 1142-1146. doi: 10.1023/A:1023781132498.

[27] Bruguera M, Bertran A, Bombi JA, Rodes J: Giant mitochondria in hepatocytes: a diagnostic hint for alcoholic liver disease. Gastroenterology 1977;73:1383-1387.

[28] Junge J, Horn T, Christoffersen P: Megamitochondria as a diagnostic marker for alcohol induced centrilobular and periportal fibrosis in the liver. Virchows Arch A Pathol Anat Histopathol 1987;410:553-558.

[29] Nakano M, Worner TM, Lieber CS: Perivenular fibrosis in alcoholic liver injury: ultrastructure and histologic progression. Gastroenterology 1982;83:777-785.

[30] Edmondson HA, Peters RL, Reynolds TB, Kuzma OT: Sclerosing Hyaline Necrosis of the Liver in the Chronic Alcoholic. A Recognizable Clinical Syndrome. Ann Intern Med 1963;59:646-673. doi: 10.7326/0003-481959-5-646.

[31] Yip WW, Burt AD: Alcoholic liver disease. Semin Diagn Pathol 2006;23:149160. doi: 10.1053/j.semdp.2006.11.002.

[32] Brunt EM, Janney CG, Di Bisceglie AM, Neuschwander-Tetri BA, Bacon BR: Nonalcoholic steatohepatitis: a proposal for grading and staging the histological lesions. Am J Gastroenterol 1999;94:2467-2474. doi: 10.1111/j.1572-0241.1999.01377.x.

[33] Naveau S, Gaude G, Asnacios A, Agostini H, Abella A, Barri-Ova N, Dauvois B, Prevot S, Ngo Y, Munteanu M, Balian A, Njike-Nakseu M, Perlemuter G,
Poynard T: Diagnostic and prognostic values of noninvasive biomarkers of fibrosis in patients with alcoholic liver disease. Hepatology 2009;49:97-105. doi: $10.1002 /$ hep.22576

[34] Diehl AM, Goodman Z, Ishak KG: Alcohollike liver disease in nonalcoholics. A clinical and histologic comparison with alcohol-induced liver injury. Gastroenterology 1988;95:1056-1062.

[35] Friedrich-Rust M, Ong MF, Herrmann E, Dries V, Samaras $P$, Zeuzem $S$, Sarrazin C: Real-time elastography for noninvasive assessment of liver fibrosis in chronic viral hepatitis. AJR Am J Roentgenol 2007;188:758-764. doi: 10.2214/AJR.06.0322.

[36] Forrest EH, Gleeson D: Is a liver biopsy necessary in alcoholic hepatitis? ] Hepatol 2012;56:1427-1428. doi: 10.1016/j.jhep.2011.12.028.

[37] Dhanda AD, Collins PL, McCune CA: Is liver biopsy necessary in the management of alcoholic hepatitis? World J Gastroenterol 2013;19:78257829. doi: $10.3748 /$ wjg.v19.i44.7825.

[38] Potts JR, Verma S: Alcoholic hepatitis: diagnosis and management in 2012. Expert Rev Gastroent 2012;6:695-710. doi: 10.1586/egh.12.57.

[39] Tannapfel A, Dienes HP, Lohse AW: The indications for liver biopsy. Dtsch Arztebl Int 2012;109:477-483.

[40] Bouchier IA, Hislop WS, Prescott RJ: A prospective study of alcoholic liver disease and mortality. J Hepatol 1992;16:290-297. doi: 10.1016/S01688278(05)80659-2.

[41] Mathurin P, Duchatelle V, Ramond MJ, Degott C, Bedossa P, Erlinger S, Benhamou JP, Chaput JC, Rueff B, Poynard T: Survival and prognostic factors in patients with severe alcoholic hepatitis treated with prednisolone. Gastroenterology 1996;110:1847-1853. doi: 10.1053/gast.1996.v110. pm8964410.

[42] Beyoglu D, Idle JR: The metabolomic window into hepatobiliary disease. J Hepatol 2013;59:842-858. doi: 10.1016/j.jhep.2013.05.030. 